OPEN ACCESS

Edited by:

Carles Canto,

Nestlé Institute of Health

Sciences (NIHS), Switzerland

Reviewed by:

Peter Bai,

University of Debrecen, Hungary

Aishwarya Swaminathan,

University of Massachusetts Medical

School, United States

${ }^{*}$ Correspondence:

Marcus Buschbeck

mbuschbeck@carrerasresearch.org

Specialty section:

This article was submitted to Epigenomics and Epigenetics,

a section of the journa

Frontiers in Genetics

Received: 02 July 2018

Accepted: 06 September 2018

Published: 09 October 2018

Citation:

Hurtado-Bagès S, Guberovic I and

Buschbeck M (2018) The

MacroH2A1.1 - PARP1 Axis

at the Intersection Between Stress

Response and Metabolism

Front. Genet. 9:417.

doi: 10.3389/fgene.2018.00417

\section{The MacroH2A1.1 - PARP1 Axis at the Intersection Between Stress Response and Metabolism}

\author{
Sarah Hurtado-Bagès ${ }^{1,2}$, Iva Guberovic ${ }^{1,3}$ and Marcus Buschbeck ${ }^{1,4 *}$ \\ 1 Josep Carreras Leukaemia Research Institute, Campus ICO-Germans Trias i Pujol, Universitat Autònoma de Barcelona, \\ Badalona, Spain, ${ }^{2}$ Ph.D. Program in Biomedicine, Department of Experimental and Health Sciences, Universitat Pompeu \\ Fabra, Barcelona, Spain, ${ }^{3}$ Ph.D. Program in Biomedicine, Faculty of Pharmacy and Food Science, University of Barcelona, \\ Barcelona, Spain, ${ }^{4}$ Program for Predictive and Personalized Medicine of Cancer, Germans Trias i Pujol Research Institute \\ (PMPPC-IGTP), Badalona, Spain
}

The exchange of replication-coupled canonical histones by histone variants endows chromatin with specific features. The replacement of the canonical H2A histone for the histone variant macroH2A is one of the most remarkable epigenetic modifications. The three vertebrate macroH2A proteins have a unique tripartite structure consisting of H2A-like domain, unstructured linker, and macrodomain. Macrodomains are ancient globular folds that are able to bind nicotinamide adenine dinucleotide $\left(\mathrm{NAD}^{+}\right)$derived metabolites. Here, we will briefly describe the physiological relevance of the metabolite binding in the context of chromatin. In particular, we will focus on the macroH2A1.1 isoform that binds ADP-ribose and poly-ADP-ribose polymerase 1 (PARP1) enzyme, a cellular stress sensor. We will discuss the impact of this interaction in the context of cancer, senescence, cell stress and energy metabolism.

Keywords: epigenetic, metabolism, stress response, macroH2A1.1, PARP1

\section{INTRODUCTION}

The adaptation of cells and organisms to the environment requires coordinate and rapid response to external stimuli and stress. In order to do so, throughout evolution cells developed complex molecular mechanisms that directly influence gene regulation without changing the DNA sequence. These mechanisms, also called epigenetic regulation, modify the chromatin composition and structure. The exchange of replication-coupled histones for histone variants is one of the major chromatin modifications. Histone variants differ from replication-coupled canonical histones in their protein structure, timing of expression, and genomic distribution (Buschbeck and Hake, 2017). Depending on the cellular context, they are incorporated into chromatin in a locusspecific manner by specialized protein chaperones and ATP-dependent chromatin remodeling enzymes. In this way, histone variant incorporation endows chromatin with particular properties. During the last years, it has become clear that histone variants play key roles in epigenetically regulated processes such as development, cancer, and somatic cell reprogramming (Buschbeck and Hake, 2017). Within a diverse group of $\mathrm{H} 2 \mathrm{~A}$ variants, macroH2As are the most divergent group of histone variants with a unique tripartite structure (Buschbeck and di Croce, 2010). 
The hallmark of macroH2A structure is its evolutionary conserved C-terminal macrodomain. Intriguingly, this macrodomain mediates the interaction of the macroH2A1.1 isoform with poly-ADP-ribose polymerase 1 (PARP1) enzyme, which is a nuclear stress sensor (Kustatscher et al., 2005). Here, we will discuss the effect of this interaction on cell physiology with a particular focus on stress response and cell metabolism. We believe that a better understanding of the macroH2A1.1-PARP1 axis will help to interpret reported lossand gain-of-function phenotypes.

\section{PRESENTING THE PLAYERS}

\section{MacroH2A1.1 - A Structural Chromatin Component Able to Bind ADP-Ribose}

MacroH2A histone variants are highly conserved since the emergence of multicellular life (Rivera-Casas et al., 2016). They are widely distributed across the genome and make up approximately $1 \%$ of the total $\mathrm{H} 2 \mathrm{~A}$ pool (Buschbeck and Hake, 2017). In mammals, two genes, H2AFY and H2AFY2, encode for macroH2A1 and macroH2A2, respectively (Pehrson and Fried, 1992; Costanzi and Pehrson, 2001). Alternative splicing of macroH2A1 further gives rise to macroH2A1.1 and macroH2A1.2 isoforms (Pehrson et al., 1997). MacroH2As are unique for their atypical tripartite protein structure comprised of an N-terminal H2A-like domain, which is fused by an unstructured linker region to a large C-terminal macrodomain (Chakravarthy et al., 2005; Kustatscher et al., 2005). It was recently demonstrated that macroH2A plays a major structural role in the maintenance of heterochromatin architecture and nuclear organization (Fu et al., 2015; Douet et al., 2017). While macroH2A has a role in transcriptional repression (Costanzi and Pehrson, 1998; Mermoud et al., 1999), it is also involved in signal-induced gene activation (discussed in Creppe et al., 2012) and in suppressing transcriptional noise (Lavigne et al., 2015). In this way, macroH2A directly or indirectly affects transcriptional regulation in an ambivalent manner.

Loss-of-function studies have shown that macroH2A promotes differentiation of embryonic and adult stem cells (Pasque et al., 2012) and inhibits somatic cell reprogramming (Pasque et al., 2011). While macroH2As contribute to the robustness of embryonic development in zebrafish (Buschbeck et al., 2009), mice lacking a single macroH2A gene develop without overt developmental defects and are viable and fertile (Changolkar et al., 2007; Boulard et al., 2010). On the other hand, mice lacking both macroH2A-encoding genes are growth retarded (Pehrson et al., 2014). In cancer, macroH2A1.1 and macroH2A2 mostly act as tumor suppressors, while the role of macroH2A1.2 seems to be largely context- and cell typedependent (Cantariño et al., 2013; Corujo and Buschbeck, 2018).

It remains unclear if the macrodomain of macroH2A is involved in the general function of the protein. Crystal structures of macroH2A macrodomains revealed the presence of a binding pocket within all isoforms. Nevertheless, these binding pockets differ significantly in size and hydrophobicity (Kustatscher et al., 2005). Generally, macrodomains can be found across most species (Rack et al., 2016). They are ancient globular domains that are able to bind ADP-ribose moieties and derivatives (reviewed in Posavec et al., 2013). Ladurner group made a pioneering discovery showing that macroH2A1.1 macrodomain also specifically binds nicotinamide adenine dinucleotide $\left(\mathrm{NAD}^{+}\right)$derived metabolites such as ADP-ribose and $O$-acetyl-ADP-ribose (Kustatscher et al., 2005). This binding of ADP-ribose enables macroH2A1.1 to interact with activated PARP1 enzyme (Karras et al., 2005; Timinszky et al., 2009). On the other hand, macroH2A1.2 and macroH2A2 isoforms are unable to bind these metabolites.

\section{PARP1 - A Major Nuclear Stress Sensor of the Cell}

Poly-ADP-ribose polymerases, also known as diphteria toxin-like ADP-ribosyltransferases (ARTDs), are a conserved superfamily of enzymes present in all domains of life, from bacteria to human (Citarelli et al., 2010; Daugherty et al., 2014). In mammals, up to 17 PARP genes share specific signature motifs in the catalytic domain (Luo and Kraus, 2012). PARPs are stress sensor enzymes that are best studied in the context of DNA damage repair (reviewed in Amours et al., 1999), transcription, and chromatin organization (reviewed in Kraus and Lis, 2003). On the wholecell level, PARPs regulate several cellular processes including proliferation and differentiation, apoptosis, pro-inflammatory responses, neuronal long-term memory, mitochondrial function, and metabolic stress (for reviews, please see Chiarugi, 2002; Schreiber et al., 2006; Languren et al., 2013; Vida et al., 2017).

Poly-ADP-ribose polymerase 1 is the founding and the best characterized member of the PARP superfamily (Ame et al., 1999). It is highly abundant in eukaryote nuclei and one of three nuclear PARPs that are activated by discontinuous DNA structures caused by DNA damage. Indeed, PARP1 is implicated in the repair of single-strand or double-strand breaks either by homologous recombination or non-homologous end joining (reviewed in Rouleau et al., 2010). When activated, PARP1 hydrolyzes $\mathrm{NAD}^{+}$generating ADP-ribose moieties (reviewed in Schreiber et al., 2006). Consequently, PARP1 transfers this newly formed ADP-ribose to target proteins resulting in mono- or polyADP-ribosylation (MARylation and PARylation, respectively). The main targets of activated PARP1 are histones and PARP1 itself, as a consequence of auto-PARylation (reviewed in Kim et al., 2005).

Poly-ADP-ribose polymerase 1 is composed of three functional domains, namely, N-terminal DNA-binding domain, central auto-modification domain, and C-terminal catalytic domain (Langelier et al., 2012). The binding to damaged DNA mediates conformational change of PARP1 increasing its affinity for $\mathrm{NAD}^{+}$and leading to the activation of PARP1 catalytic domain. PAR formation has at least three functions: (i) creation of a platform for the recruitment of effector proteins including the DNA repair machinery, (ii) disruption of protein-protein or protein-nucleic acid interactions leading 
to chromatin relaxation, and (iii) generation of signal for protein ubiquitination and degradation (reviewed in Gibson and Kraus, 2012). Auto-PARylation of PARP1 reduces its affinity toward DNA creating a negative feedback loop which inhibits its own activity (Zahradka and Ebisuzaki, 1982). Removal of PARP1 from DNA and rapid enzymatic turnover of PAR are essential for optimal function of the DNA repair machinery (reviewed in Rouleau et al., 2010). Interestingly, it has been reported that PARP1 can also be activated in a DNA-independent manner through the interaction with ERK2 (extracellular signalregulated kinase 2) (Cohen-Armon et al., 2007). Therefore, this may implicate PARP enzymes in other cellular processes unrelated to DNA, opening new lines of research in the field of PARP.

In vivo studies showed that mice lacking PARP1 are hypersensitive to genotoxic agents or $\gamma$-irradiation, demonstrating the importance of PARP1 for efficient DNA repair (Ménissier-de Murcia, 1997; Wang et al., 1997). Pharmacological inhibition of PARP1 was first introduced in cancer therapy to exploit synthetic lethality of BRCA1-deficient breast tumors (Fong et al., 2009) and is now widely tested in combination with DNA damaging chemotherapeutic drugs (Farmer et al., 2005). In addition to cancer therapy, PARP inhibitors may have additional benefits in other diseases such as cardiovascular or metabolic disorders (Pacher and Szabo, 2007; Shevalye et al., 2010).

\section{Physiological Studies Link MacroH2A1 and PARP1 to Metabolism}

Systemic loss-of-function studies in mice linked both macroH2A1 and PARP1 to metabolic phenotypes, although some observations are controversial and sometimes lead to opposite conclusions. Here, we summarize and discuss some of these findings (for a more comprehensive view, please see Table 1).

Although mice lacking macroH2As display only mild phenotypes, they repeatedly exhibit metabolic disorders. Indeed, macroH2A1 knockout (KO) mice displayed impaired clearance of bolus glucose injections, suggesting a pre-diabetic phenotype and partial insulin resistance (Changolkar et al., 2007). However, when fed with high-fat diet, increased energy expenditure was observed and was accompanied by lower accumulation of fat and increased leanness (Sheedfar et al., 2015). Furthermore, deregulation of genes involved in lipid metabolism was reported in the liver of mice from different genetic backgrounds (Changolkar et al., 2007; Boulard et al., 2010). However, liver fat accumulation was only observed in female mice with $50 \%$ penetrance (Boulard et al., 2010). Liver cancer cell lines showed to be protected from lipid accumulation upon overexpression of macroH2A1.1 (Pazienza et al., 2014). Moreover, overexpression of macroH2A1.2 in mice was found to reduce adipose tissue and thus to promote leanness (Pazienza et al., 2016). In summary, macroH2A has been reported as a metabolic regulator with opposing effects in mice. Such discrepancies might be explained by the use of mice with different genetic backgrounds, ages or genders and distinct outcomes can be observed depending on the macroH2A isoforms depleted (Table 1).
Similarly to macroH2A1, contradictory results have been observed with PARP1 KO mice, displaying improved or deteriorated metabolic fitness (reviewed in Bai and Cantó, 2012; Table 1). On the one hand, mice lacking PARP1 displayed aberrant circadian rhythms (Asher et al., 2010) and diet-induced obesity (Devalaraja-Narashimha and Padanilam, 2010) associated with hepatic steatosis (Erener et al., 2012a). On the other hand, PARP1 inhibition and deletion in mice were associated with improvement of diabetes- or obesity-related parameters such as glucose tolerance, weight loss, or fat mass reduction (reviewed in Bai and Cantó, 2012; Table 1). Furthermore, PARP1 depletion is correlated with improved mitochondrial and heart function (Wen et al., 2018). Plethora of studies showed that PARP1 can contribute to both gene activation and repression. For example, its interaction with metabolic regulatory transcription factors, such as FOXO1 (Sakamaki et al., 2009) and PPAR $\gamma$ (Erener et al., 2012a), affects genes implicated in mitochondrial respiration and fatty acid oxidation (Bai et al., 2011).

In a living system, different tissues can affect each other's homeostasis and therefore give rise to differential physiological phenotypes. This could explain the conflicting results observed in macroH2A1 and PARP1 KO mice. Despite discrepancies, the majority of studies suggest unfavorable effects of PARP1 enzyme on metabolism. On the other hand, macroH2A1.1 is often associated with beneficial metabolic outcomes (Table 1).

\section{THE MACROH2A1.1-PARP1 AXIS}

Although macroH2A and PARP proteins have been studied for decades, the physiological consequences and mechanisms of their interaction are still unclear and require further research. Depending on the relative abundance of the two proteins and the strength of PARP1 activity, their interaction may have at least two different outcomes. On the one hand, macroH2A1.1 can recruit active PARP1 to chromatin and thus act in concert to regulate gene expression. On the other hand, macroH2A1.1 can bind and inhibit PARP1 activity, with global consequences on cellular metabolism. Although an interconnection between macroH2A1.1 and PARP1 was also observed in the context of DNA damage response (Timinszky et al., 2009), the molecular mechanism of such processes needs to be further investigated.

\section{The Effect on Gene Regulation}

MacroH2A1.1 and PARP1 cooperate to repress and activate genes in response to external signals. This is best understood in the context of the transcriptional regulation of the heat shock-inducible Hsp70.1 gene. In HeLa cells, exogenous macroH2A1.1 was found to recruit PARP1 to the silenced Hsp70.1 promoter (Ouararhni et al., 2006). Heat shock activates PARP1 resulting in significant auto-PARylation and PARylation of target proteins. This leads to the release of both macroH2A1.1 and PARP-1 from chromatin, and as consequence activates the Hsp70.1 gene (Ouararhni et al., 2006). In fibroblasts, the interaction of macroH2A1.1 with activated PARP1 contributes to both positive and negative gene 


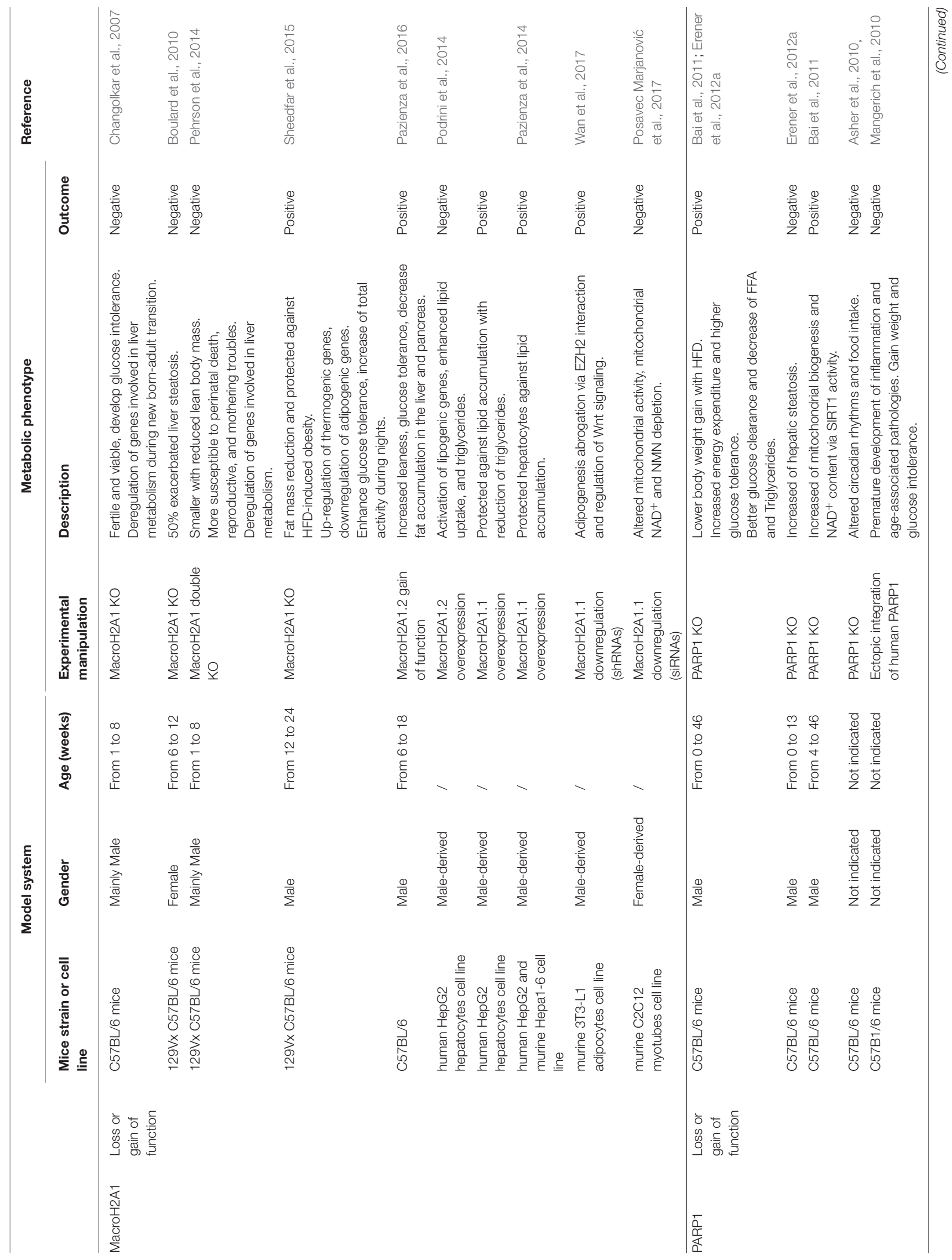




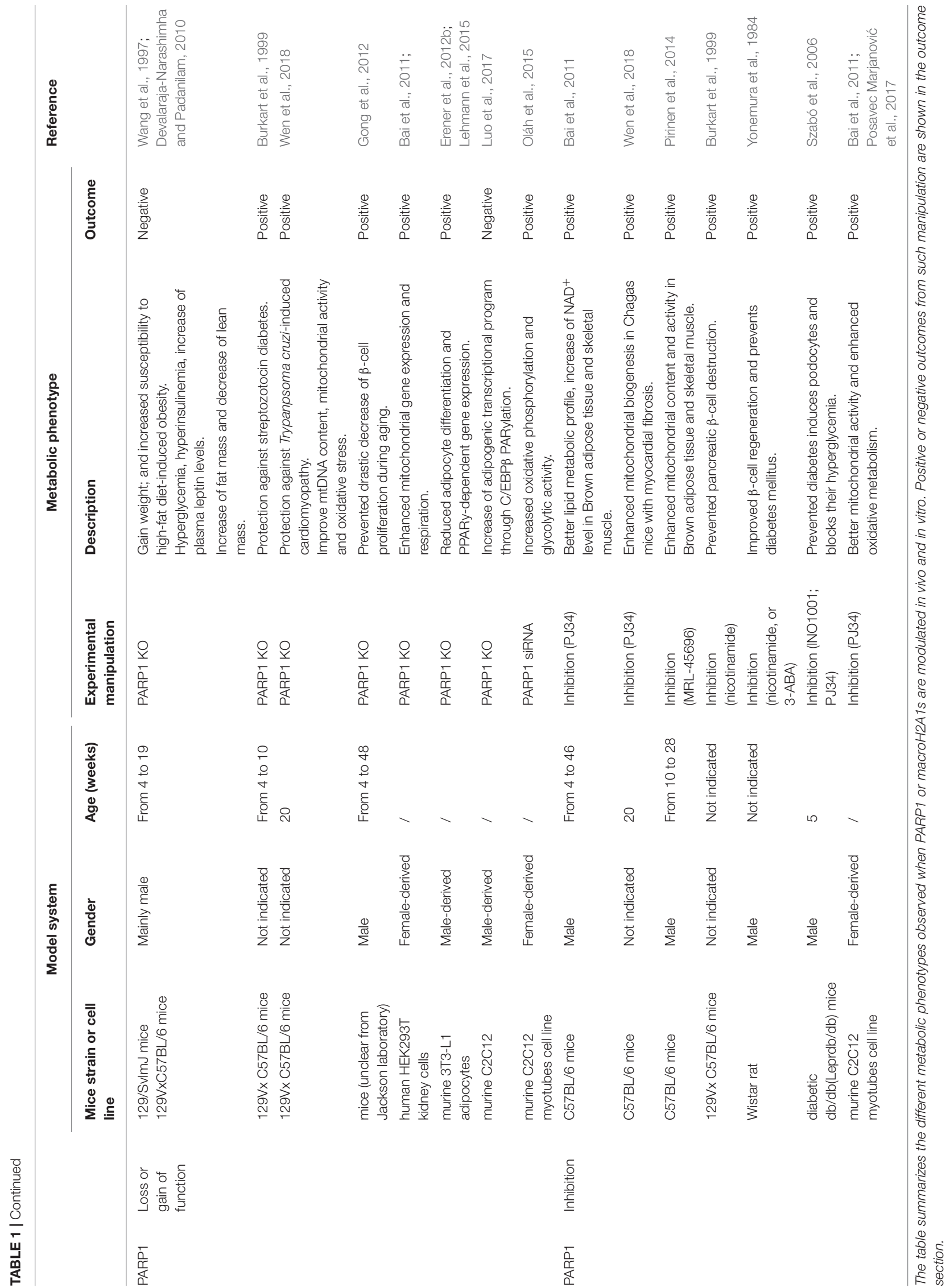


regulation (Chen et al., 2014). Mechanistically, macroH2A1.1bound PARP1 recruits the histone acetyltransferase CBP promoting $\mathrm{H} 2 \mathrm{~B}$ acetylation of its target genes (Chen et al., 2014). Furthermore, macroH2A1.1-PARP1 axis is important for the regulation of SASP (senescence-associated secretory phenotype) genes in cancer cell model (Chen et al., 2015). Additionally, macroH2A1.1 facilitates differentiation of 3T3-L1 preadipocytes by inhibiting regulatory genes such as Wnt10b (Wan et al., 2017). This phenotype was only observed with macroH2A1.1 isoform suggesting the potential implication of macroH2A1.1-PARP1 axis in adipogenesis. In summary, macroH2A1.1-PARP1 axis regulates cellular stress response in a transcription-dependent manner (Figure 1). Consequently, it affects several physiological outcomes such as cancer, senescence, and possibly adipogenesis.

\section{The Effect on NAD ${ }^{+}$Metabolism}

Beside gene regulation, the macroH2A1.1-PARP1 axis was reported to influence cellular $\mathrm{NAD}^{+}$pools. $\mathrm{NAD}^{+}$is a wellknown coenzyme essential for redox reactions in metabolism. Beyond its crucial role in glycolysis and mitochondrial respiration, $\mathrm{NAD}^{+}$is also involved in the regulation of gene expression, DNA repair, calcium signaling, circadian rhythms, lifespan, and cell death (reviewed in Cantó et al., 2015). The maintenance of $\mathrm{NAD}^{+}$levels is mainly ensured through its salvage which requires much fewer enzymatic

\section{Stress Signal}
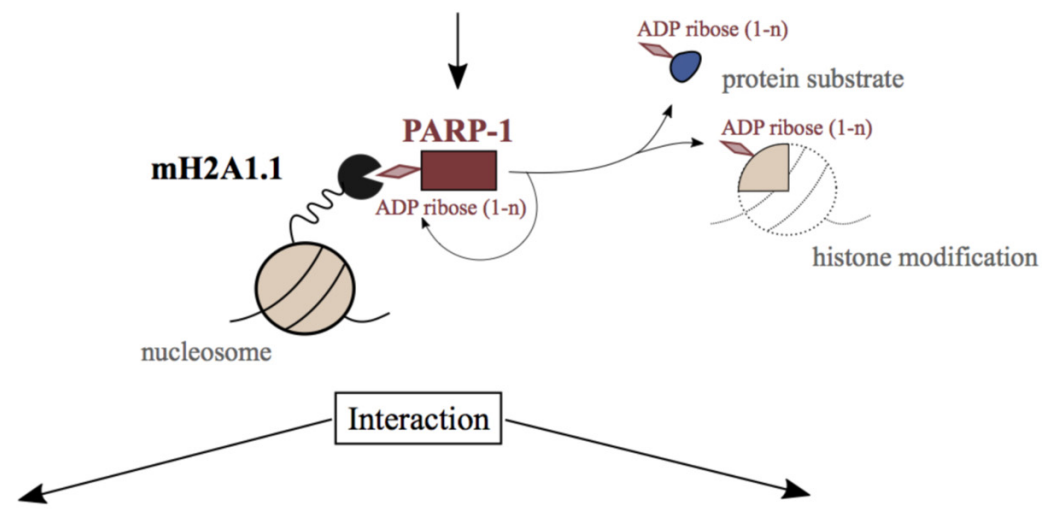

High level of mH2A1.1

PARP-1 inhibition

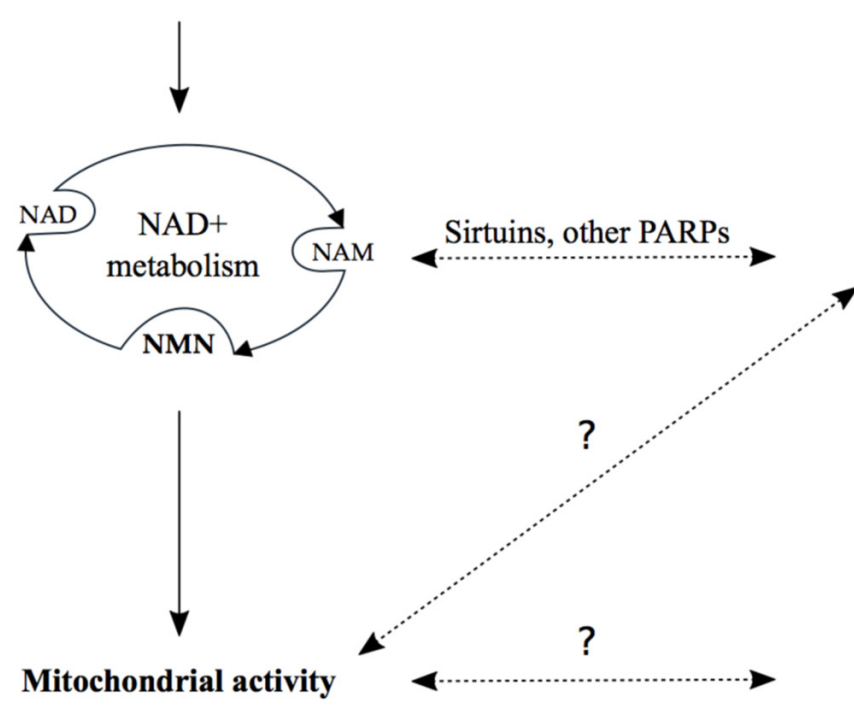

High level of activated PARP1

PARP-1 recruitement

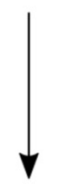

Gene regulation

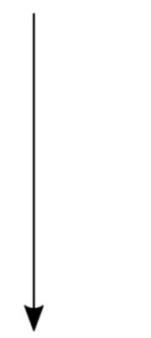

Stress response

FIGURE 1 | Physiological function of the MacroH2A1.1_PARP1 axis. Stressing signals generated during DNA damage repair, senescence, hormonal response, heat shock, or differentiation promote the binding of macroH2A1.1 to activated PARP1 creating the macroH2A1.1-PARP1 axis. On the one hand, when macroH2A1.1 is highly expressed, this interaction leads to the inhibition of PARP1 activity which reduces PARP1 consumption of nuclear NAD+. In this way, the macroH2A1.1-PARP1 axis influences mitochondrial activity through subcellular NAD pools turnover. On the other hand, macroH2A1.1 and active PARP1 act in concert in order to regulate gene expression and as a consequence regulate stress signals response. Crosstalks between those both pathways implicating sirtuin and other PARP enzymes may additionally influence the physiological outcomes of the macroH2A1.1-PARP1 axis. The question marks illustrate the lack of knowledge regarding some steps in this axis pathway. 
reactions than its de novo synthesis from dietary sources (reviewed in Verdin, 2015). The consumption of $\mathrm{NAD}^{+}$by PARPs and sirtuin deacetylases (SIRTs) generates ADP-ribose and NAM (nicotinamide). NAM is subsequently converted into nicotinamide mononucleotide $(\mathrm{NMN})$, which is a major $\mathrm{NAD}^{+}$ precursor (Figure 1). Interestingly, more than 50 years ago, it was shown that NMN addition to liver nuclear extract stimulates PARylation supporting the link between $\mathrm{NAD}^{+}$salvage pathway and PARP1 activity (Chambon et al., 1963). Since then, NAD ${ }^{+}$ and its salvage pathway have been demonstrated to be essential for nuclear, cytoplasmic, and mitochondrial activities.

A breakthrough appeared with the development of molecular tools able to detect free $\mathrm{NAD}^{+}$(Cambronne et al., 2016) and ATP (Imamura et al., 2009) in sub-cellular compartments. This allowed for monitoring of how the imbalance of $\mathrm{NAD}^{+}$and ATP in one organelle affects the whole cellular metabolism (Wright et al., 2016; Ryu et al., 2018). Among all PARPs, PARP1 is the major $\mathrm{NAD}^{+}$consumer in the nucleus (reviewed in Fouquerel and Sobol, 2014). Prolonged PARP1 activation leads to depletion of cellular NAD ${ }^{+}$and ATP (Hassa et al., 2006). As a consequence, this leads to a misbalance of sub-cellular $\mathrm{NAD}^{+}$pools, which results in mitochondrial dysfunction (Virág et al., 1998a; Cipriani et al., 2005), increased oxidative stress and cell death (Virág et al., 1998b; Zong et al., 2004).

Proliferative cells have high nuclear energy demand and mainly use glycolysis to ensure fast synthesis of nucleic acids. On the other hand, terminally differentiated cells rather rely on mitochondrial respiration in order to maintain tissue functions (Leary et al., 1998). In this respect, in commonly used cell model for skeletal muscle differentiation, it was shown that the macroH2A1.1-PARP1 axis protected differentiated myotubes from oxidative stress (Oláh et al., 2015; Posavec Marjanović et al., 2017). Indeed, a decrease of PARP1 protein level and activity was observed during muscle differentiation which was associated with higher resistance to oxidative damage (Bai et al., 2011; Szczesny et al., 2013; Oláh et al., 2015). Furthermore, a splicing switch of macroH $2 \mathrm{~A} 1$ occurs during cell differentiation, which results in the increase of macroH2A1.1 levels (Sporn and Jung, 2012; Posavec Marjanović et al., 2017). Additionally, our group observed that high abundance of macroH2A1.1 leads to the reduction of nuclear consumption of $\mathrm{NAD}^{+}$by PARP1 in differentiated myotubes. In this way, macroH2A1.1 maintains optimal $\mathrm{NAD}^{+}$and NMN levels in the different cellular compartments, thereby facilitating proper respiratory capacity of mitochondria (Posavec Marjanović et al., 2017). Interestingly, dietary supplementation with NMN enhanced mitochondrial oxidative metabolism in mice which was associated with the suppression of ageassociated body weight (Yeung et al., 2016). Following a similar trend, pharmacologic inhibition of PARP1 or its genetic

\section{REFERENCES}

Ame, J., Rolli, V., Schreiber, V., Niedergang, C., Apiou, F., Decker, P., et al. (1999). PARP-2, a novel mammalian DNA damage-dependent poly(ADP-ribose) polymerase. J. Biol. Chem. 274, 17860-17868. doi: 10.1074/jbc.274.25.17860 deletion improves mitochondrial function (Pirinen et al., 2014). This happens due to the increase of $\mathrm{NAD}^{+}$availability, which promotes SIRT1 activation and downstream regulation of metabolic genes (Bai et al., 2011). In conclusion, the macroH2A1.1-PARP1 axis can influence $\mathrm{NAD}^{+}$metabolism and consequentially the activity of distant organelles, such as mitochondria (Figure 1). This can occur independently of gene regulation or could involve other $\mathrm{NAD}^{+}$sensitive transcriptional processes.

\section{CONCLUSION AND FUTURE PERSPECTIVES}

MacroH2As are commonly studied with respect to their function as transcriptional regulators and epigenome stabilizers. Intriguingly, recent results highlighted a new function of macroH2A1.1, which is to establish adequate threshold for PARP1 activity and help maintain cell energy homeostasis. Future work will be necessary to better understand how different stress signals affect the macroH2A1.1-PARP1 axis and a possible involvement of other partners, such as SIRTs or other PARPs. This new discovery redefines research boundaries of macroH2A field and raises the need to investigate the function of macroH2A considering its effects on both nuclear and whole-cell level. In conclusion, the study of the macroH2A1.1PARP1 axis could have a great clinical relevance, especially in diseases related to $\mathrm{NAD}^{+}$deficiency, such as sarcopenia and pellagra.

\section{AUTHOR CONTRIBUTIONS}

SH-B, IG, and MB wrote this review.

\section{FUNDING}

$\mathrm{SH}-\mathrm{B}$ is enrolled in the Ph.D. program in Biomedicine of the University Pompeu Fabra and IG in the Ph.D. program in Biomedicine of the University of Barcelona. IG was supported by the Marie Skłodowska Curie Training network "ChroMe" (H2020-MSCA-ITN-2015-675610). Research in the Buschbeck lab was further supported by grants from MINECO-ISCIII (PIE16/00011), MINECO (BFU2015-66559-P), the Deutsche Jose Carreras Leukaemie Stiftung, and AGAUR (2017-SGR-305). Research at the IJC was supported by the "La Caixa" Foundation, the Fundació Internacional Josep Carreras, Celgene Spain, and the CERCA Program/Generalitat de Catalunya.

Amours, D. D., Desnoyers, S., D’Silva, I., and Poirier, G. G. (1999). Poly(ADPribosyl) ation reactions in the regulation of nuclear functions. Biochem. J. 342, 249-268. doi: 10.1042/bj3420249

Asher, G., Reinke, H., Altmeyer, M., Gutierrez-Arcelus, M., Hottiger, M. O., and Schibler, U. (2010). Poly-(ADP-ribose) polymerase 1 participates in the phase 
entrainment of circadian clocks to feeding. Cell 142, 943-953. doi: 10.1016/j. cell.2010.08.016

Bai, P., and Cantó, C. (2012). The role of PARP-1 and PARP-2 enzymes in metabolic regulation and disease. Cell Metab. 16, 290-295. doi: 10.1016/j.cmet.2012.06.016

Bai, P., Cantó, C., Oudart, H., Brunyánszki, A., Cen, Y., Thomas, C., et al. (2011). PARP-1 inhibition increases mitochondrial metabolism through SIRT1 activation. Cell Metab. 13, 461-468. doi: 10.1016/j.cmet.2011.03.004

Boulard, M., Storck, S., Cong, R., Pinto, R., Delage, H., and Bouvet, P. (2010). Histone variant macroH2A1 deletion in mice causes female-specific steatosis. Epigenetics Chromatin 3:8. doi: 10.1186/1756-8935-3-8

Burkart, V., Wang, Z.-Q., Radons, J., Heller, B., Herceg, Z., Stingl, L., et al. (1999). Mice lacking the poly(ADP-ribose) polymerase gene are resistant to pancreatic beta-cell destruction and diabetes development induced by streptozocin. Nat. Med. 5, 314-319. doi: 10.1038/6535

Buschbeck, M., and di Croce, L. (2010). Approaching the molecular and physiological function of macroH2A variants. Epigenetics 5, 118-123. doi: 10. 4161/epi.5.2.11076

Buschbeck, M., and Hake, S. B. (2017). Variants of core histones and their roles in cell fate decisions, development and cancer. Nat. Rev. Mol. Cell Biol. 18, 299-314. doi: 10.1038/nrm.2016.166

Buschbeck, M., Uribesalgo, I., Wibowo, I., Rué, P., Martin, D., Gutierrez, A., et al. (2009). The histone variant macroH2A is an epigenetic regulator of key developmental genes. Nat. Struct. Mol. Biol. 16, 1074-1079. doi: 10.1038/nsmb. 1665

Cambronne, X. A., Stewart, M. L., Kim, D., Jones-Brunette, A. M., Morgan, R. K., Farrens, D. L., et al. (2016). Biosensor reveals multiple sources for mitochondrial $\mathrm{NAD}^{+}$. Science $352,1474-1477$. doi: $10.1126 /$ science.aad5168

Cantariño, N., Douet, J., and Buschbeck, M. (2013). MacroH2A - an epigenetic regulator of cancer. Cancer Lett. 336, 247-252. doi: 10.1016/j.canlet.2013.03.022

Cantó, C., Menzies, K. J., and Auwerx, J. (2015). NAD ${ }^{+}$metabolism and the control of energy homeostasis: a balancing act between mitochondria and the nucleus. Cell Metab. 22, 31-53. doi: 10.1016/j.cmet.2015.05.023

Chakravarthy, S., Gundimella, S. K., Caron, C., Perche, P. Y., Pehrson, J. R., Khochbin, S., et al. (2005). Structural characterization of the histone variant macroH2A. Mol. Cell. Biol. 25, 7616-7624. doi: 10.1128/MCB.25.17.7616-7624. 2005

Chambon, P., Weill, J. D., and Mandel, P. (1963). Nicotinamide mononucleotide activation of a new DNA-dependent polyadenylic acid synthesizing nuclear enzyme. Biochem. Biophys. Res. Commun. 11, 39-43. doi: 10.1016/0006291X(63)90024-X

Changolkar, L. N., Costanzi, C., Leu, N. A., Chen, D., McLaughlin, K. J., Pehrson, J. R., et al. (2007). Developmental changes in histone macroH2A1mediated gene regulation. Mol. Cell. Biol. 27, 2758-2764. doi: 10.1128/MCB. 02334-06

Chen, H., Ruiz, P. D., McKimpson, W. M., Novikov, L., Kitsis, R. N., and Gamble, M. J. (2015). MacroH2A1 and ATM play opposing roles in paracrine senescence and the senescence-associated secretory phenotype. Mol. Cell 59, 719-731. doi: 10.1016/j.molcel.2015.07.011

Chen, H., Ruiz, P. D., Novikov, L., Casill, A. D., Park, J. W., and Gamble, M. J. (2014). MacroH2A1.1 and PARP-1 cooperate to regulate transcription by promoting CBP-mediated H2B acetylation. Nat. Struct. Mol. Biol. 21, 981-989. doi: $10.1038 / \mathrm{nsmb} .2903$

Chiarugi, A. (2002). Poly(ADP-ribose) polymerase: killer or conspirator? The "suicide hypothesis" revisited. Trends Pharmacol. Sci. 23, 122-129. doi: 10.1016/ S0165-6147(00)01902-7

Cipriani, G., Rapizzi, E., Vannacci, A., Rizzuto, R., Moroni, F., and Chiarugi, A. (2005). Nuclear poly(ADP-ribose) polymerase-1 rapidly triggers mitochondrial dysfunction. J. Biol. Chem. 280, 17227-17234. doi: 10.1074/jbc.M414526200

Citarelli, M., Teotia, S., and Lamb, R. S. (2010). Evolutionary history of the poly(ADP-ribose) polymerase gene family in eukaryotes. BMC Evol. Biol. 10:308. doi: 10.1186/1471-2148-10-308

Cohen-Armon, M., Visochek, L., Rozensal, D., Kalal, A., Geistrikh, I., Klein, R., et al. (2007). DNA-independent PARP-1 activation by phosphorylated ERK2 increases Elk1 activity: a link to histone acetylation. Mol. Cell 25, 297-308. doi: 10.1016/j.molcel.2006.12.012

Corujo, D., and Buschbeck, M. (2018). Post-translational modifications of H2A histone variants and their role in cancer. Cancers 10, 1-25. doi: 10.3390/ cancers 10030059
Costanzi, C., and Pehrson, J. R. (1998). Histone macroH2A1 is concentrated in the inactive X chromosome of female mammals. Nature 628, 1997-1999. doi: $10.1038 / 31275$

Costanzi, C., and Pehrson, J. R. (2001). MACROH2A2, a new member of the MACROH2A core histone family. J. Biol. Chem. 276, 21776-21784. doi: 10.1074/jbc.M010919200

Creppe, C., Posavec, M., Douet, J., and Buschbeck, M. (2012). MacroH2A in stem cells: a story beyond gene repression. Epigenomics 4, 221-227. doi: 10.2217/ epi.12.8

Daugherty, M. D., Young, J. M., Kerns, J. A., and Malik, H. S. (2014). Rapid evolution of PARP genes suggests a broad role for ADP-ribosylation in hostvirus conflicts. PLoS Genet. 10:e1004403. doi: 10.1371/journal.pgen.1004403

Devalaraja-Narashimha, K., and Padanilam, B. J. (2010). PARP1 deficiency exacerbates diet-induced obesity in mice. J. Endocrinol. 205, 243-252. doi: 10.1677/JOE-09-0402

Douet, J., Corujo, D., Malinverni, R., Renauld, J., Sansoni, V., Marjanović, M. P., et al. (2017). MacroH2A histone variants maintain nuclear organization and heterochromatin architecture. J. Cell Sci. 130, 1570-1582. doi: 10.1242/jcs. 199216

Erener, S., Hesse, M., Kostadinova, R., and Hottiger, M. O. (2012a). Poly(ADPRibose)polymerase-1 (PARP1) controls adipogenic gene expression and adipocyte function. Mol. Endocrinol. 26, 79-86. doi: 10.1210/me.2011-1163

Erener, S., Mirsaidi, A., Hesse, M., Tiaden, A. N., Ellingsgaard, H., Kostadinova, R., et al. (2012b). ARTD1 deletion causes increased hepatic lipid accumulation in mice fed a high-fat diet and impairs adipocyte function and differentiation. FASEB J. 26, 2631-2638. doi: 10.1096/fj.11-200212

Farmer, H., McCabe, N., Lord, C. J., Tutt, A. N., Johnson, D. A., Richardson, T. B., et al. (2005). Targeting the DNA repair defect in BRCA mutant cells as a therapeutic strategy. Nature 434, 917-921. doi: 10.1038/nature03445

Fong, P. C., Boss, D. S., Yap, T. A., Tutt, A., Wu, P., Mergui-Roelvink, M., et al. (2009). Inhibition of poly(ADP-Ribose) polymerase in tumors from BRCA mutation carriers. N. Engl. J. Med. 362, 1597-1605. doi: 10.1056/ NEJMoa1215817

Fouquerel, E., and Sobol, R. W. (2014). ARTD1 (PARP1) activation and $\mathrm{NAD}^{+}$in DNA repair and cell death. DNA Repair 23, 27-32. doi: 10.1016/j.dnarep.2014. 09.004

Fu, Y., Yan, G., Fan, H., Cheng, L., Zhang, F., Dang, Y., et al. (2015). MacroH2A1 associates with nuclear lamina and maintains chromatin architecture in mouse liver cells. Sci. Rep. 5:17186. doi: 10.1038/srep17186

Gong, L., Liu, F.-q., Wang, Y., Hou, X.-g., Zhang, W., Qin, W.-d., et al. (2012). Poly (ADP-Ribose) transferase/polymerase-1-deficient mice resistant to agedependent decrease in $\beta$-cell proliferation. Mol. Med. 18, 816-824. doi: 10.2119/ molmed.2011.00458

Gibson, B. A., and Kraus, W. L. (2012). New insights into the molecular and cellular functions of poly(ADP-ribose) and PARPs. Nat. Rev. Mol. Cell Biol. 13, 411-424. doi: $10.1038 / \mathrm{nrm} 3376$

Hassa, P. O., Haenni, S. S., Elser, M., and Hottiger, M. O. (2006). Nuclear ADPribosylation reactions in mammalian cells: where are we today and where are we going? Microbiol. Mol. Biol. Rev. 70, 789-829. doi: 10.1128/MMBR. 00040-05

Imamura, H., Nhat, K. P., Togawa, H., Saito, K., Iino, R., Kato-Yamada, Y., et al. (2009). Visualization of ATP levels inside single living cells with fluorescence resonance energy transfer-based genetically encoded indicators. Proc. Natl. Acad. Sci. U.S.A. 106, 15651-15656. doi: 10.1073/pnas.0904764106

Karras, G. I., Kustatscher, G., Buhecha, H. R., Allen, M. D., Pugieux, C., Sait, F., et al. (2005). The macro domain is an ADP-ribose binding module. EMBO J. 24, 1911-1920. doi: 10.1038/sj.emboj.7600664

Kim, M. Y., Zhang, T., and Kraus, W. L. (2005). Poly (ADP-ribosyl) ation by PARP-1: PAR-laying NAD into a nuclear signal. Genes Dev. 19, 1951-1967. doi: $10.1101 /$ gad.1331805.ity

Kraus, W. L., and Lis, J. T. (2003). PARP goes transcription. Cell 113, 677-683. doi: 10.1016/S0092-8674(03)00433-1

Kustatscher, G., Hothorn, M., Pugieux, C., Scheffzek, K., and Ladurner, A. G. (2005). Splicing regulates NAD metabolite binding to histone macroH2A. Nat. Struct. Mol. Biol. 12, 624-625. doi: 10.1038/nsmb956

Langelier, M. F., Planck, J. L., Roy, S., and Pascal, J. M. (2012). Structural basis for DNA damage-dependent poly(ADP-ribosyl)ation by human PARP-1. Science 336, 728-732. doi: $10.1126 /$ science. 1216338 
Languren, G., Montiel, T., Julio-Amilpas, A., and Massieu, L. (2013). Neuronal damage and cognitive impairment associated with hypoglycemia: an integrated view. Neurochem. Int. 63, 331-343. doi: 10.1016/j.neuint.2013.06.018

Lavigne, M. D., Vatsellas, G., Polyzos, A., Mantouvalou, E., Sianidis, G., Maraziotis, I., et al. (2015). Composite macroH2A/NRF-1 nucleosomes suppress noise and generate robustness in gene expression. Cell Rep. 11, 1090-1101. doi: 10.1016/j.celrep.2015.04.022

Leary, S. C., Battersby, B. J., Hansford, R. G., and Moyes, C. D. (1998). Interactions between bioenergetics and mitochondrial biogenesis. Biochim. Biophys. Acta 1365, 522-530. doi: 10.1016/S0005-2728(98)00105-4

Lehmann, M., Pirinen, E., Mirsaidi, A., Kunze, F. A., Richards, P. J., Auwerx, J., et al. (2015). ARTD1-induced poly-ADP-ribose formation enhances PPAR $\gamma$ ligand binding and co-factor exchange. Nucleic Acids Res. 43, 129-142. doi: $10.1093 /$ nar/gku1260

Luo, X., and Kraus, W. L. (2012). On PAR with PARP: cellular stress signaling through poly(ADP-ribose) and PARP-1. Genes Dev. 26, 417-432. doi: 10.1101/ gad.183509.111

Luo, X., Ryu, K. W., Kim, D.-S., Nandu, T., Medina, C. J., Gupte, R., et al. (2017). PARP-1 controls the adipogenic transcriptional program by PARylating C/EBP $\beta$ and modulating its transcriptional activity. Mol. Cell 65, 260-271. doi: 10.1016/j.molcel.2016.11.015

Mangerich, A., Herbach, N., Hanf, B., Fischbach, A., Popp, O., Moreno-Villanueva, M., et al. (2010). Inflammatory and age-related pathologies in mice with ectopic expression of human PARP-1. Mech. Ageing Dev. 131, 389-404. doi: 10.1016/j. mad.2010.05.005

Ménissier-de Murcia, J. (1997). Requirement of poly ( ADP-ribose ) polymerase in recovery from DNA damage in mice and in cells. Proc. Natl. Acad. Sci. U.S.A. 94, 7303-7307. doi: 10.1073/pnas.94.14.7303

Mermoud, J. E., Costanzi, C., Pehrson, J. R., and Brockdorff, N. (1999). Histone MacroH2A1.2 relocates to the inactive $\mathrm{X}$ chromosome after initiation and propagation of X-inactivation. J. Cell Biol. 147, 1399-1408. doi: 10.1083/jcb.147. 7.1399

Oláh, G., Szczesny, B., Brunyánszki, A., López-García, I. A., Gerö, D., Radák, Z., et al. (2015). Differentiation-associated downregulation of poly(ADP-Ribose) polymerase-1 expression in myoblasts serves to increase their resistance to oxidative stress. PLoS One 10:e134227. doi: 10.1371/journal.pone.01 34227

Ouararhni, K., Hadj-Slimane, R., Ait-Si-Ali, S., Robin, P., Mietton, F., HarelBellan, A., et al. (2006). The histone variant mH2A1. 1 interferes with transcription by down-regulating PARP-1 enzymatic activity. Genes Dev. 20, 3324-3336. doi: 10.1101/gad.396106.essential

Pacher, P., and Szabo, C. (2007). Role of poly(ADP-ribose) polymerase 1 (PARP-1) in Cardiovasc, cardiovascular diseases: the therapeutic potential of PARP inhibitors. Cardiovasc. Drug Rev. 25, 235-260. doi: 10.1111/j.1527-3466.2007. 00018.x

Pasque, V., Gillich, A., Garrett, N., and Gurdon, J. B. (2011). Histone variant macroH2A confers resistance to nuclear reprogramming. EMBO J. 30, 2373-2387. doi: 10.1038/emboj.2011.144

Pasque, V., Radzisheuskaya, A., Gillich, A., Halley-Stott, R. P., Panamarova, M., and Zernicka-Goetz, M. (2012). Histone variant macroH2A marks embryonic differentiation in vivo and acts as an epigenetic barrier to induced pluripotency. J. Cell Sci. 125, 6094-6104. doi: 10.1242/jcs.113019

Pazienza, V., Borghesan, M., Mazza, T., Sheedfar, F., Panebianco, C., Williams, R., et al. (2014). SIRT1-metabolite binding histone MacroH2A1.1 protects hepatocytes against lipid accumulation. Aging 6, 35-47. doi: 10.18632/aging. 100632

Pazienza, V., Panebianco, C., Rappa, F., Memoli, D., Borghesan, M., Cannito, S., et al. (2016). Histone MacroH2A1.2 promotes metabolic health and leanness by inhibiting adipogenesis. Epigenetics Chromatin 9, 1-19. doi: 10.1186/s13072016-0098-9

Pehrson, J. R., Changolkar, L. N., Costanzi, C., and Leu, N. A. (2014). Mice without MacroH2A histone variants. Mol. Cell. Biol. 34, 4523-4533. doi: 10.1128/MCB. 00794-14

Pehrson, J. R., Costanzi, C., and Dharia, C. (1997). Developmental and tissue expression patterns of histone macroH2A1 subtypes. J. Cell. Biochem. 65, 107-113. doi: 10.1002/(SICI)1097-4644(199704)65:1<107::AID-JCB11>3.0. $\mathrm{CO} ; 2-\mathrm{H}$
Pehrson, J. R., and Fried, V. A. (1992). MacroH2A, a core histone containing a large nonhistone region. Science 257, 1398-1400. doi: 10.1126/science.1529340

Pirinen, E., Cantó, C., Jo, Y. S., Morato, L., Zhang, H., Menzies, K. J., et al. (2014). Pharmacological inhibition of poly(ADP-ribose) polymerases improves fitness and mitochondrial function in skeletal muscle. Cell Metab. 19, 1034-1041. doi: 10.1016/j.cmet.2014.04.002

Podrini, C., Koffas, A., Chokshi, S., Vinciguerra, M., Lelliott, C. J., White, J. K., et al. (2014). MacroH2A1 isoforms are associated with epigenetic markers for activation of lipogenic genes in fat-induced steatosis. FASEB J. 29, 1676-1687. doi: 10.1096/fj.14-262717

Posavec, M., Timinszky, G., and Buschbeck, M. (2013). Macro domains as metabolite sensors on chromatin. Cell. Mol. Life Sci. 70, 1509-1524. doi: 10. 1007/s00018-013-1294-4

Posavec Marjanović, M., Hurtado-Bagès, S., Lassi, M., Valero, V., Malinverni, R., Delage, H., et al. (2017). MacroH2A1.1 regulates mitochondrial respiration by limiting nuclear $\mathrm{NAD}^{+}$consumption. Nat. Struct. Mol. Biol. 24, 902-910. doi: $10.1038 / \mathrm{nsmb} .3481$

Rack, J. G. M., Perina, D., and Ahel, I. (2016). Macrodomains: structure, function, evolution, and catalytic activities. Annu. Rev. Biochem. 85, 431-454. doi: 10. 1146/annurev-biochem-060815-014935

Rivera-Casas, C., Gonzalez-Romero, R., Cheema, M. S., Ausió, J., and Eirín-López, J. M. (2016). The characterization of macroH2A beyond vertebrates supports an ancestral origin and conserved role for histone variants in chromatin. Epigenetics 11, 415-425. doi: 10.1080/15592294.2016.1172161

Rouleau, M., Patel, A., Hendzel, M. J., Kaufmann, S. H., and Poirier, G. G. (2010). PARP inhibition: PARP1 and beyond. Nat. Rev. Cancer 10, 293-301. doi: $10.1038 / \mathrm{nrc} 2812$

Ryu, K. W., Nandu, T., Kim, J., Challa, S., DeBerardinis, R. J., and Kraus, W. L. (2018). Metabolic regulation of transcription through compartmentalized $\mathrm{NAD}^{+}$biosynthesis. Science 360:eaan5780. 5doi: 10.1126/science.aan 5780

Sakamaki, J., Daitoku, H., Yoshimochi, K., Miwa, M., and Fukamizu, A. (2009). Regulation of FOXO1-mediated transcription and cell proliferation by PARP-1. Biochem. Biophys. Res. Commun. 382, 497-502. doi: 10.1016/j.bbrc.2009.03.022

Schreiber, V., Dantzer, F., Ame, J. C., and de Murcia, G. (2006). Poly(ADP-ribose): novel functions for an old molecule. Nat. Rev. Mol. Cell Biol. 7, 517-528. doi: $10.1038 / \mathrm{nrm} 1963$

Sheedfar, F., Vermeer, M., Pazienza, V., Villarroya, J., Rappa, F., Cappello, F., et al. (2015). Genetic ablation of macrohistone H2A1 leads to increased leanness, glucose tolerance and energy expenditure in mice fed a high-fat diet. Int. J. Obes. 39, 331-338. doi: 10.1038/ijo.2014.91

Shevalye, H., Maksimchyk, Y., Watcho, P., and Obrosova, I. G. (2010). Poly(ADPribose) polymerase-1 (PARP-1) gene deficiency alleviates diabetic kidney disease. Biochim. Biophys. Acta 1802, 1020-1027. doi: 10.1016/j.bbadis.2010. 07.004

Sporn, J. C., and Jung, B. (2012). Differential regulation and predictive potential of MacroH2A1 isoforms in colon cancer. Am. J. Pathol. 180, 2516-2526. doi: 10.1016/j.ajpath.2012.02.027

Szabó, C., Biser, A., Benko, R., Böttinger, E., and Suszták, K. (2006). Poly(ADPribose) polymerase inhibitors ameliorate nephropathy of type 2 diabetic Leprdb/db mice. Diabetes 55, 3004-3012. doi: 10.2337/db06-0147

Szczesny, B., Olah, G., Walker, D. K., Volpi, E., Rasmussen, B. B., Szabo, C., et al. (2013). Deficiency in repair of the mitochondrial genome sensitizes proliferating myoblasts to oxidative damage. PLoS One 8:e75201. doi: 10.1371/ journal.pone.0075201

Timinszky, G., Till, S., Hassa, P. O., Hothorn, M., Kustatscher, G., Nijmeijer, B., et al. (2009). A macrodomain-containing histone rearranges chromatin upon sensing PARP1 activation. Nat. Struct. Mol. Biol. 16, 923-929. doi: 10.1038/ nsmb.1664

Verdin, E. (2015). $\mathrm{NAD}^{+}$in aging, metabolism, and neurodegeneration. Science 350, 1208-1213. doi: 10.1126/science.aac4854

Vida, A., Márton, J., Mikó, E., and Bai, P. (2017). Metabolic roles of poly(ADPribose) polymerases. Semin. Cell Dev. Biol. 63, 135-143. doi: 10.1016/j.semcdb. 2016.12.009

Virág, L., Salzman, A. L., and Szabo, C. (1998a). Poly(ADP-ribose) synthetase activation mediates mitochondrial injury during oxidant-induced cell death. J. Immunol. 161, 3753-3759. 
Virág, L., Scott, G. S., Cuzzocrea, S., Marmer, D., Salzman, A. L., and Szabó, C. (1998b). Peroxynitrite-induced thymocyte apoptosis: the role of caspases and poly (ADP-ribose) synthetase (PARS) activation. Immunology 94, 345-355.

Wan, D., Liu, C., Sun, Y., Wang, W., Huang, K., and Zheng, L. (2017). MacroH2A1.1 cooperates with EZH2 to promote adipogenesis by regulating Wnt signaling. J. Mol. Cell Biol. 9, 325-337. doi: 10.1093/jmcb/mjx027

Wang, Z. Q., Stingl, L., Morrison, C., Jantsch, M., Los, M., Schulze-Osthoff, K., et al. (1997). PARP is important for genomic stability but dispensable in apoptosis. Genes Dev. 11, 2347-2358. doi: 10.1101/gad.11.18.2347

Wen, J. J., Yin, Y. W., and Garg, N. J. (2018). PARP1 depletion improves mitochondrial and heart function in Chagas disease: effects on POLG dependent mtDNA maintenance. PLoS Pathog. 14:e1007065. doi: 10.1371/ journal.ppat.1007065

Wright, R. H. G., Lioutas, A., Le Dily, F., Soronellas, D., Pohl, A., Bonet, J., et al. (2016). ADP-ribose-derived nuclear ATP synthesis by NUDIX5 is required for chromatin remodeling. Science 352, 1221-1225. doi: 10.1126/science. aad 9335

Yeung, M. Y., Ding, Q., Brooks, C. R., Xiao, S., Workman, C. J., Vignali, D. A., et al. (2016). Long-term administration of nicotinamide mononucleotide mitigates age-associated physiological decline in mice. Cell Metab. 15, 942-953.
Yonemura, Y., Takashima, T., Miwa, K., Miyazaki, I., Yamamoto, H., and Okamoto, H. (1984). Amelioration of diabetes mellitus in partially depancreatized rats by poly(ADP-ribose) synthetase inhibitors: evidence of islet B-cell regeneration. Diabetes 33, 401-404. doi: 10.2337/diab.33.4.401

Zahradka, P., and Ebisuzaki, K. (1982). A shuttle mechanism for DNA-protein interactions. Eur. J. Biochem. 127, 579-585. doi: 10.1111/j.1432-1033.1982. tb06912.x

Zong, W. X., Ditsworth, D., Bauer, D. E., Wang, Z. Q., and Thompson, C. B. (2004). Alkylating DNA damage stimulates a regulated form of necrotic cell death. Genes Dev. 18, 1272-1282. doi: 10.1101/gad.1199904

Conflict of Interest Statement: The authors declare that the research was conducted in the absence of any commercial or financial relationships that could be construed as a potential conflict of interest.

Copyright (c) 2018 Hurtado-Bagès, Guberovic and Buschbeck. This is an open-access article distributed under the terms of the Creative Commons Attribution License (CC BY). The use, distribution or reproduction in other forums is permitted, provided the original author(s) and the copyright owner(s) are credited and that the original publication in this journal is cited, in accordance with accepted academic practice. No use, distribution or reproduction is permitted which does not comply with these terms. 